\title{
Contributions of the medial temporal lobe to declarative memory retrieval: Manipulating the amount of contextual retrieval
}

\author{
Indira Tendolkar, ${ }^{1,2,6}$ Jennifer Arnold, ${ }^{1,2}$ Karl Magnus Petersson, ${ }^{2}$ Susanne Weis, ${ }^{3}$ \\ Anke Brockhaus-Dumke, ${ }^{4}$ Philip van Eijndhoven, ${ }^{1,2}$ Jan Buitelaar, ${ }^{1}$ \\ and Guillén Fernández ${ }^{2,5}$ \\ ${ }^{1}$ Department of Psychiatry at Radboud University Medical Center, Nijmegen 6500-HB, The Netherlands; ${ }^{2}$ F.C. Donders \\ Centre of Cognitive Neuroimaging at Radboud University Nijmegen, Nijmegen 6500-HB, The Netherlands; ${ }^{3}$ Department \\ of Neurology, University of Aachen, Aachen 52074, Germany; ${ }^{4}$ Department of Psychiatry, University of Cologne, \\ Cologne 50924, Germany; ${ }^{5}$ Department of Neurology at Radboud University Medical Center, \\ Nijmegen 6500-HB, The Netherlands
}

\begin{abstract}
We investigated how the hippocampus and its adjacent mediotemporal structures contribute to contextual and noncontextual declarative memory retrieval by manipulating the amount of contextual information across two levels of the same contextual dimension in a source memory task. A first analysis identified medial temporal lobe (MTL) substructures mediating either contextual or noncontextual retrieval. A linearly weighted analysis elucidated which MTL substructures show a gradually increasing neural activity, depending on the amount of contextual information retrieved. A hippocampal engagement was found during both levels of source memory but not during item memory retrieval. The anterior MTL including the perirhinal cortex was only engaged during item memory retrieval by an activity decrease. Only the posterior parahippocampal cortex showed an activation increasing with the amount of contextual information retrieved. If one assumes a roughly linear relationship between the blood-oxygenation level-dependent (BOLD) signal and the associated cognitive process, our results suggest that the posterior parahippocampal cortex is involved in contextual retrieval on the basis of memory strength while the hippocampus processes representations of item-context binding. The anterior MTL including perirhinal cortex seems to be particularly engaged in familiarity-based item recognition. If one assumes departure from linearity, however, our results can also be explained by one-dimensional modulation of memory strength.
\end{abstract}

Declarative memory supports discrimination between previously encountered and novel stimuli by means of recollection and familiarity: While recollection involves recognition, including the retrieval of contextual information, familiarity refers to a feeling that a stimulus has been encountered previously without contextual retrieval (Mandler 1980).

Since Scoville and Milner (1957) reported their initial findings from patient $\mathrm{HM}$, at least 50 years of converging evidence from human and animal lesion studies, as well as neuroimaging research, have identified the medial temporal lobe (MTL) as a crucial mediator of declarative memory (for recent reviews, see Diana et al. 2007; Eichenbaum et al. 2007). Based on cytoarchitecture and anatomical connectivity, MTL substructures in humans are dissociated into the hippocampus and the parahippocampal gyrus covered by the ento- and perirhinal cortex anteriorly, and the parahippocampal cortex posteriorly, which also extends into the medial regions of the fusiform gyrus (Insausti et al. 1987).

To date, the precise contribution of the hippocampus and the perirhinal and parahippocampal cortices remains a topic of debate: Based on behavioral data from patients with selective lesions of MTL substructures, one line of research proposes that the hippocampus is critical for recollection of episodic memories,

\footnotetext{
${ }^{6}$ Corresponding author.
}

E-mail i.tendolkar@psy.umcn.nl; fax 0031-24-3540561.

Article is online at http://www.learnmem.org/cgi/doi/10.1101//m.916708. whereas the perirhinal cortex supports recognition judgments based upon familiarity (e.g., Brown and Aggleton 2001; Holdstock et al. 2005; Bowles et al. 2007; Sauvage et al. 2008). Another line of research does not support dichotomies between the functions of the hippocampus and the adjacent medial temporal structures, instead suggesting a functionally unified memory system within the MTL (e.g., Manns et al. 2003; Stark and Squire 2003; Wais et al. 2006; for a review, see Squire et al. 2004).

In recent years, functional magnetic resonance neuroimaging (fMRI) studies in healthy humans have gone beyond the natural limitations of lesion studies but have also generated controversial results. For example, while several studies report no difference between brain activity related to contextual and noncontextual memory retrieval within the MTL (e.g., Stark and Squire 2003; Kirwan and Stark 2004; Squire et al. 2004), other studies have revealed a dissociation between a hippocampal contribution to recollection (e.g., Eldridge et al. 2000; Yonelinas et al. 2001, 2005; Cansino et al. 2002; Giovanello et al. 2004; Weis et al. 2004a; Daselaar et al. 2006) and anterior MTL (most likely perirhinal) contributions to noncontextual item recognition based on familiarity (Henson et al. 2003, 2005; Weis et al. 2004a; Gonsalves et al. 2005; Daselaar et al. 2006; Montaldi et al. 2006).

Recently, the available evidence has been taken to suggest a dissociation between hippocampal and perirhinal functions and also the role of posterior parahippocampal cortex in declarative memory retrieval (Diana et al. 2007; Eichenbaum et al. 2007; see also Davachi 2006). Eichenbaum and colleagues (2007; see also Diana et al. 2007) suggested that perirhinal cortex is involved in 
familiarity-based representation of item information, while the posterior parahippocampal cortex is involved in representation of context, suggesting an important role in recollection. According to this model, the hippocampus integrates or binds the item and contextual information, and this will lead to reactivation of associated information in parahippocampal cortex during memory retrieval. Another perspective (Squire et al. 2007) suggests that different medial temporal processes are distinct but do not dissociate along the line between acontextual item memory and contextual-associative memory. In contrast, this perspective discusses a one-dimensional modulation of memory strength explaining differential behavior of MTL substructures. To explain functional imaging findings that do not show a monotonic change in activity paralleling monotonic changes in memory strength, Squire and colleagues (2007) propose a departure from linearity in the relationship between the blood-oxygenation level-dependent (BOLD) signal and memory strength in these MTL substructures.

Thus far, most of the studies have focused on contrasting one condition yielding contextual retrieval with another condition demanding simple recognition of item information (e.g., Eldridge et al. 2000; Cansino et al. 2002; Dobbins et al. 2003; Bunge et al. 2004; Giovanello et al. 2004; Weis et al. 2004a; Meltzer and Constable 2005). Such an approach is useful in dissociating the specific role of hippocampus and its adjacent mediotemporal structures in contextual and noncontextual forms of declarative memory. A complementary approach varies the amount of contextual information across several levels and allows us to investigate the neural response as a function of these levels as well as the sensitivity with which the different MTL structures react to the quantitative changes in the amount of contextual retrieval, thereby also taking into account the aspect of memory strength.

We, therefore, conducted an fMRI experiment, in which subjects were asked to remember photos of either landscapes or buildings that were dyed in different colors and different shades of these colors. In other words, the amount of contextual information was manipulated across two levels of the same contextual dimension (color) that were tested by means of a source memory task. We assumed that trials with mere item recognition reflect the least amount of contextual retrieval, trials in which retrieval of global source information (i.e., photos + color retrieval) was successful consist of an intermediate amount of contextual information, and trials in which two levels of source memory (i.e., photos + color + shade retrieval) were accomplished contain the greatest amount of contextual information. By comparing neural activity between trials reflecting successful source memory (collapsed over the two levels of contextual information) with trials reflecting simple recognition of previously presented stimuli as well as the latter ones with trials to previously presented but unrecognized stimuli (misses), we should be able to dissociate MTL activity mediating contextual and noncontextual retrieval as done previously. In particular, we were interested in whether all MTL subregions contribute equally to contextual- and non- contextual memory retrieval or not. Based on the aforementioned hypotheses (Diana et al. 2007; Eichenbaum et al. 2007), we predicted that activation of the hippocampus and the posterior parahippocampal cortex co-occur during contextual retrieval, while anterior MTL regions (most probably within the perirhinal cortex) are selectively engaged in familiarity-based recognition. Additionally, our experimental design allowed us to test how activations change depending on the retrieval of different amounts of contextual information.

In addition, by contrasting trials of old items in a linearly weighted manner (i.e., unrecognized old items $<$ recognized items $<$ recognized items for which one level of source was correctly recollected $<$ recognized items for which both levels of source were correctly recollected), we aimed to elucidate which MTL substructures responded in a gradually increasing or decreasing manner. FMRI activity in these substructures might thus reflect monotonically increasing or decreasing memory strength (Squire et al. 2007).

\section{Results}

Overall recognition was high (80.5\%). The performance rate of correct judgments for " 2 source hits," i.e., trials in which two levels of source memory (i.e., photos + color + shade retrieval) were accomplished $\left(t_{19}=8.68, P<0.001\right)$, and " 1 source hits," i.e., trials in which retrieval of global source information (i.e., photos + color retrieval) was successful $\left(t_{19}=6.62, P<0.001\right)$, differed significantly from chance. There was a better performance for 1 source hits in comparison with 2 source hits $\left(t_{19}=24.7, P<0.001\right)$, indicating that the latter one was more difficult to retrieve. For additional information also on the reaction times, see Table 1.

The global analysis (thresholded at $P=0.001$; cluster-size test statistic, corrected for multiple nonindependent comparisons) confirmed previous findings (e.g., Weis et al. 2004a,b; Hornberger et al. 2006) of a significant difference between the neural activity for all recognized items versus misses, as well as in comparison to new items in mediotemporal, parietal, and prefrontal regions (for details, see Tables 2, 3). To delineate the involvement of the MTL substructures, a subsequent region of interest (ROI) analysis was performed within the MTL, as outlined in the Materials and Methods section (cf., Tendolkar et al. 2007).

By means of $t$-tests, we investigated whether there were any significant differences between hits with correct retrieval of context (collapsed over 1 source hits and 2 source hits) and hits without correct retrieval of context ("item hits"), as well as significant differences between hits with correct retrieval of context (collapsed over 1 source hits and 2 source hits) and correctly classified new items ("correct rejections"). We also included an analysis of activity decreases and increases between "misses" and item hits as well as correct rejections and item hits to further dissociate neural activity related to mere item recognition (Henson et al. 2003, 2005; Weis et al. 2004a; Gonsalves et al. 2005; Daselaar et al. 2006; Montaldi et al. 2006).

Table 1. Behavioral results

\begin{tabular}{|c|c|c|c|c|c|c|c|c|}
\hline & \multicolumn{5}{|c|}{ Old items } & \multicolumn{3}{|c|}{ New items } \\
\hline & Uncertain & Misses & 2 source hits & 1 source hits & Item hits & Uncertain & Correct rejection & False alarms \\
\hline $\begin{array}{l}\% \text { of trials } s^{a} \\
\text { No. of trials } \\
\text { Reaction times }\end{array}$ & $\begin{aligned} 6.2 & \pm 2.6 \\
15 & \pm 6 \\
2100 & \pm 440\end{aligned}$ & $\begin{aligned} 13.4 & \pm 3 \\
32 & \pm 7 \\
2076 & \pm 443\end{aligned}$ & $\begin{aligned} 23.6 & \pm 5 \\
57 & \pm 12 \\
1943 & \pm 411\end{aligned}$ & $\begin{aligned} 27.2 & \pm 4.2 \\
65 & \pm 14 \\
2003 & \pm 434\end{aligned}$ & $\begin{aligned} 29.6 & \pm 7.4 \\
71 & \pm 18 \\
2061 & \pm 474\end{aligned}$ & $\begin{aligned} 6.7 & \pm 2.1 \\
8 & \pm 3 \\
2122 & \pm 484\end{aligned}$ & $\begin{aligned} 72.8 & \pm 7.4 \\
87 & \pm 9 \\
1828 & \pm 381\end{aligned}$ & $\begin{aligned} 20.5 & \pm 4.5 \\
25 & \pm 5 \\
1948 & \pm 355\end{aligned}$ \\
\hline
\end{tabular}

${ }^{\mathrm{a}}$ Mean percentage of trials ( \pm standard deviation).

${ }^{b}$ Mean number of trials ( \pm standard deviation).

cMean reaction times ( \pm standard deviation). 
An fMRI study of declarative memory retrieval

Table 2. Brain regions showing significantly greater BOLD signal $(P<0.05$, corrected at the cluster level) during the overall analysis for recognized than for unrecognized stimuli during retrieval

\begin{tabular}{|c|c|c|c|c|c|c|}
\hline \multirow[b]{2}{*}{ Region } & \multirow[b]{2}{*}{ Left/right } & \multirow[b]{2}{*}{ No. of voxels $/ \mathrm{mm}^{3}$} & \multicolumn{3}{|c|}{ MNI coordinates } & \multirow[b]{2}{*}{ Z-value } \\
\hline & & & $x$ & $Y$ & $Z$ & \\
\hline Inferior frontal gyrus & Left & $16,628 / 715,004$ & -44 & 4 & 30 & 6.4 \\
\hline Caudate nucleus & Left & $2389 / 102,727$ & -12 & 10 & 0 & 6.04 \\
\hline Medial frontal gyrus & Left & $152 / 6536$ & -24 & 32 & -16 & 4.45 \\
\hline Parahippocampal gyrus & Right & $38 / 1634$ & 3 & -26 & -26 & 3.53 \\
\hline
\end{tabular}

Second, we conducted an analysis testing how the activation pattern of the MTL substructures changes depending on the retrieval of different amounts of contextual information. The mean activation for all old items were taken and linearly weighted depending on the amount of contextual retrieval or memory strength that was associated with each condition (i.e., 2 source hits $>1$ source hits $>$ item hits $>$ misses).

Below we will report the results of these analyses arranged by MTL substructures.

\section{Hippocampus}

The first analysis showed a larger hippocampal activity (cluster size: 20 voxels $/ 860 \mathrm{~mm}^{3}$ ) for hits with correct contextual retrieval (collapsed over 1 source hits and 2 source hits) compared with those without (item hits) with local maxima in the middle $(-28$, $\left.-26,-10 ; P_{\text {corr }}<0.01\right)$ and the dorsal aspect of the left hippocampus $\left(-30,-38,0 ; P_{\text {corr }}<0.01\right)$ (Fig. $\left.1 \mathrm{~A}\right)$. In order to dissociate the hippocampal activity that was selectively engaged by either of the two source memory conditions, we compared directly 1 source hits and 2 source hits. While the comparison 2 source hits versus 1 source hits gave rise to a larger activation in the left middle/anterior hippocampus (cluster size: 8 voxels $/ 344 \mathrm{~mm}^{3}$; $\left.-24,-28,-10 ; P_{\text {corr }}<0.001\right), 1$ source hits versus 2 source hits did not reveal any significant hippocampal activation (Fig. 2). Moreover, no hippocampal activation was found for noncontextual memory when comparing item hits with either misses or correct rejections. Finally, the linearly weighted analysis did not show any significant effects within the hippocampus.

\section{Anterior MTL}

In a region of anterior MTL (Fig. 1B), which includes the perirhinal cortex, the first analysis gave rise to a decrease of activity for item hits opposed to misses (cluster size: 6 voxels $/ 258 \mathrm{~mm}^{3} ; 24$, $-2,-30 ; P_{\text {corr }}<0.05$ ) and opposed to correct rejections (cluster size: 5 voxels $/ 215 \mathrm{~mm}^{3} ; 28,0,-26 ; P_{\text {corr }}<0.05$ ). None of the other contrasts as outlined previously showed any significant effects within this region.

\section{Posterior parahippocampal cortex}

Analyses revealed no significant activations when contrasting hits with correct retrieval of context (collapsed over 1 source hits and 2 source hits) to those without (item hits) as well as correctly classified new items (correct rejections). There was significantly more activity for item hits compared with misses in left fusiform gyrus most probably covered by parahippocampal cortex (cluster size: 9 voxels $/ 387 \mathrm{~mm}^{3} ;-32,-42,-20 ; P_{\text {corr }}<0.05$ ). However, no significant difference between item hits and correct rejections were found. Finally, the linearly weighted analysis (i.e., 2 source hits $>1$ source hits $>$ item hits $>$ misses) showed significant effects within the left (cluster size: 10 voxels $/ 430 \mathrm{~mm}^{3} ;-20,-44$, $\left.-6 ; P_{\text {corr }}<0.01\right)$ and the right posterior parahippocampal cortex (cluster size: 18 voxels $/ 774 \mathrm{~mm}^{3} ; 24,-56,-2 ; P_{\text {corr }}<0.05$ ). Indeed, the bar graphs in Figure $1 \mathrm{C}$ depicting the contrast estimates show the graded nature of this memory effect.

\section{Discussion}

In this study, we investigated how the hippocampus and its adjacent mediotemporal cortices contribute to contextual and noncontextual forms of declarative memory retrieval. A hippocampal engagement could only be identified during contextual-memory retrieval, while activity in anterior MTL was only found in the form of an activity decrease related to noncontextual item recognition. Posterior parahippocampal cortex showed an activation gradually increasing with the amount of contextual information retrieved.

First, our results replicate earlier fMRI studies showing a selective engagement of the hippocampus in contextual memory retrieval (Eldridge et al. 2000; Cansino et al. 2002; Giovanello et al. 2004; Weis et al. 2004a; Daselaar et al. 2006; for review, see Eichenbaum et al. 2007), thereby supporting a specific role for the hippocampus in recollective aspects of declarative memory (Brown and Aggleton 2001; Eichenbaum and Cohen 2001; O'Reilly and Norman 2002). Moreover, recollection of both levels of source memory compared with just one level resulted in a more anterior extension of the hippocampal activation. In this context, we note that the hippocampus receives input originating from diverse neocortical regions. The neocortex and the hippocampus are indirectly connected via the parahippocampal gyrus, whereby anterior neocortical regions connect to the anterior parahippocampal cortex, and whereby posterior neocortical regions connect to the posterior parahippocampal cortex (Insausti et al. 1987; Suzuki and Amaral 1994). The increase of activation pattern along the longitudinal axis of the hippocampus in our study might thus reflect an increase in hippocampal input related to the retrieval of additional contextual information from different neocortical sites. However, it is rather unlikely that color and shade of a color induce different neocortical representations. Hence, it is more likely that the extension of hippocam-

Table 3. Brain regions showing significantly greater BOLD signal $(P<0.05$, corrected at the cluster level) during the overall analysis for recognized than for correctly rejected new stimuli during retrieval

\begin{tabular}{|c|c|c|c|c|c|c|}
\hline \multirow[b]{2}{*}{ Region } & \multirow[b]{2}{*}{ Left/right } & \multirow[b]{2}{*}{ No. of voxels $/ \mathrm{mm}^{3}$} & \multicolumn{3}{|c|}{ MNI coordinates } & \multirow[b]{2}{*}{ Z-value } \\
\hline & & & $x$ & $Y$ & $Z$ & \\
\hline $\begin{array}{l}\text { Supplementary motor area } \\
\text { Inferior parietal gyrus } \\
\text { Precuneus }\end{array}$ & $\begin{array}{l}\text { Left } \\
\text { Left } \\
\text { Left }\end{array}$ & $\begin{array}{c}6844 / 294,292 \\
2912 / 125,216 \\
921 / 39,603\end{array}$ & $\begin{array}{r}-4 \\
-28 \\
-8\end{array}$ & $\begin{array}{r}10 \\
-62 \\
-68\end{array}$ & $\begin{array}{l}52 \\
42 \\
54\end{array}$ & $\begin{array}{l}7.5 \\
6.5 \\
5.4\end{array}$ \\
\hline
\end{tabular}



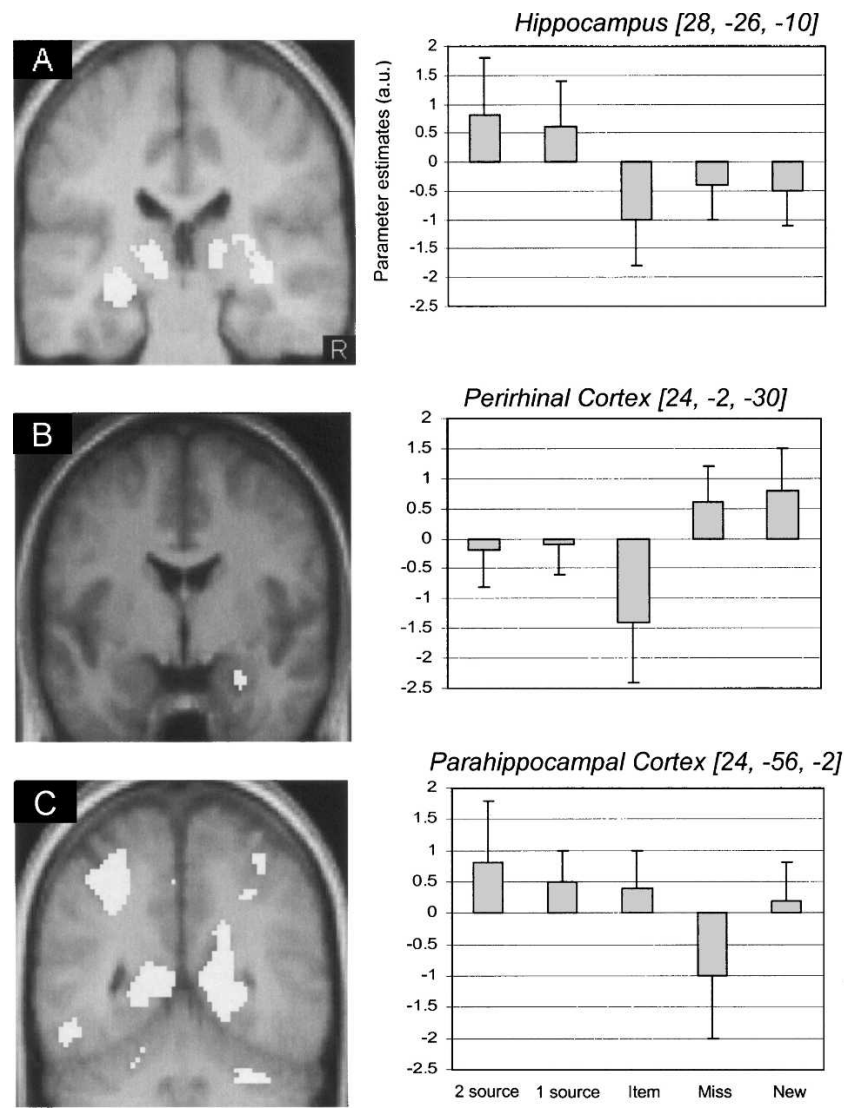

Figure 1. Specific activations from the ROI analyses within the MTL ( $P_{\text {corrected }}<0.05$, thresholded at a voxel size of 10 ) of the hippocampus $(A)$, right anterior MTL $(B)$, and left and right posterior parahippocampal cortex $(C)$ are shown superimposed onto selected coronal slices of the mean high-resolution T1-weighted volume supplied with SPM2. R refers to the right side of the brain. The bar graphs show the corresponding contrast estimates derived from the local maxima of the ROI analyses. The hippocampal activation $(A)$ reflects the stepwise contrast between hits with correct contextual retrieval (collapsed over 1 source hits and 2 source hits) compared with those without (item hits), while the anterior MTL effect with a local maximum in perirhinal cortex $(B)$ reflects the significant signal decrease of merely recognized stimuli (item hits) compared with misses and correct rejections. Finally, the posterior parahippocampal activation $(C)$ results from a linear trend analysis reflecting the different amount of contextual retrieval across all four conditions (2 source hits, 1 source hits, item hits, misses) of previously presented stimuli as outlined in the Materials and Methods section.

pal activations along its longitudinal axis reflect an increase in "intrinsic" hippocampal information processing related to the retrieval of more binding or associations between the picture and its color context. As our data contain the same kind of contextual information, color, these do not speak to the question of whether the posterior or anterior parts of the hippocampus in healthy humans may be responsible for dissociable functions in contextual retrieval (Moser and Moser 1998).

The observed activation of posterior parahippocampal cortex in relation to contextual memory retrieval is also in line with several other studies (e.g., Cansino et al. 2002; Kirwan and Stark 2004; Yonelinas et al. 2005, Hayes et al. 2007). Our findings extend previous results in that we show that activation of posterior parahippocampal cortex increased as a function of the amount of contextual information that had to be reactivated for successful retrieval. Given that color and shade of color represent two features of the same contextual dimension, the gradual increase in parahippocampal cortex can best be interpreted as a parahippocampal process contributing to the reactivation of actual contextual representations during retrieval. Thus, our data are in line with the hypothesis that the posterior parahippocampal cortex contributes to the recollection of contextual information (Diana et al. 2007; Eichenbaum et al. 2007).

However, we found a monotonic increase in activity from misses over item hits and 1 source hits to 2 source hits in the parahippocampal cortex (Fig. 1C). Appreciating the results as depicted in Figure 1 and described in the Results section suggests that the monotonic increase of parahippocampal activation is driven by an increase in contextual retrieval or memory strength (Squire et al. 2007). Our data cannot discern between these two interpretations for the posterior parahippocampal activation pattern found. Note that during the memory encoding part of this study (Tendolkar et al. 2007), posterior parahippocampal activation was also found, which increased with the amount of contextual information to be encoded. Hence, converging the data from encoding and retrieval supports the idea that posterior parahippocampal cortex might be involved in both encoding and retrieval in a way that is related to memory strength or the amount of contextual information encoded/retrieved.

The hippocampal activation pattern (Fig. 1A) shows quite distinctive responses for trials with and without source retrieval, indicating a specific role in associative retrieval of source information. The pattern of hippocampal activation might also be in line with a memory strength interpretation, but only if one assumes a nonlinear relationship between the BOLD signal and memory strength as suggested by Squire and colleagues in their recent review (Squire et al. 2007). Such departure from linearity may make a dissociation of hippocampal responses to item hits and misses potentially impossible.

Finally, we found less perirhinal activity for successful item recognition compared with trials in which recognition failed or was correctly rejected as being new. Such a decrease in activity related to successful item recognition has previously been explained by a model in which new or unfamiliar items require

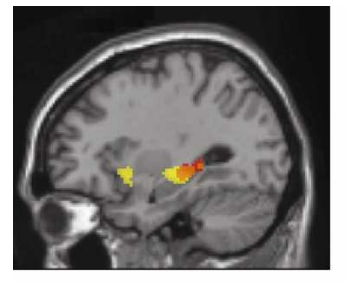

$\mathbf{x}=\mathbf{- 3 0}$

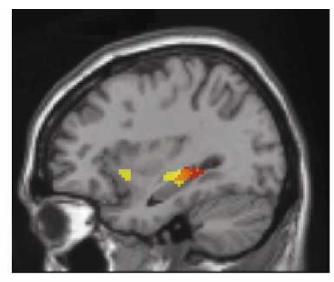

$x=-32$

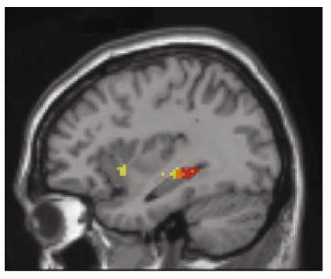

$\mathbf{x}=\mathbf{- 3 4}$

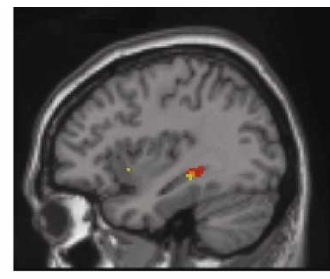

$x=-36$

Figure 2. Displayed are the results from the stepwise $t$-tests $\left(P_{\text {corrected }}<0.05\right.$, thresholded at a voxel size of 10$)$ for 2 source hits versus item hits (shown in yellow) and 1 source hits versus item hits (shown in red). The activations are displayed on sagittal slices of the standard T1-weighted volume supplied with SPM2. The activation shown in orange reflects the results from a conjunctional analysis (thresholded at $P<0.001$ uncorrected) between the hippocampal activation for 1 source hits and 2 source hits superimposed on the aforementioned stepwise analysis. 
more neural resources than familiar items (Henson et al. 2003, 2005; Weis et al. 2004a; Gonsalves et al. 2005; Daselaar et al. 2006; Montaldi et al. 2006; Hornberger et al. 2006). However, such findings have also been interpreted as being in line with a memory strength account by assuming nonlinearity in the relationship between the BOLD signal and memory strength (Squire et al. 2007). Indeed, we found that activity in the same region during memory formation increased as a function of the amount of information that was encoded (Tendolkar et al. 2007). Thus, the increase for misses during retrieval could also mean that the items might be treated as new and therefore generate encoding related activity. In other words, retrieval and encoding related activity in this MTL region might represent a correlate of the very same process, starting to encode new information and diminishing encoding when a familiarity signal is present (Fernández and Tendolkar 2006).

As discussed, the interpretation of our data hinges significantly on the assumption that there is either a linear or nonlinear relationship between the BOLD signal and the cognitive process at issue. If one assumes linearity, the parahippocampal response might be readily linked to the amount of associative information retrieved or the associated memory strength. However, perirhinal and hippocampal response patterns under this assumption more easily explained by a process dissociation between distinct processes linked either to item or associative memory. Contrary, if one assumes nonlinear process-BOLD functions with different characteristics in different MTL substructures, all our results can be interpreted as in line with a memory strength account. Several studies have shown linear relations between neural activity and associated BOLD responses in a wide range of regions, but nonlinear components occur at the extremes and these nonlinearities appear to be different in different brain structures (e.g., Boynton et al. 1996; Soltysik et al. 2004). Although there is no good reason to assume different nonlinear functions within the MTL, there is, to the best of our knowledge, no study confirming the linearity of the neural-BOLD coupling in the MTL. Hence, we cannot exclude this alternative interpretation currently.

In summary, as long as one assumes linearity between the BOLD signal and the associated cognitive process, our results suggest that the posterior parahippocampal cortex is involved in contextual retrieval, potentially on the basis of memory strength, while the hippocampus processes representations of item-context binding. The anterior MTL including the perirhinal cortex seems to be particularly engaged in familiarity-based item recognition.

\section{Materials and Methods}

After giving informed consent, 20 healthy, right-handed subjects (10 females; mean age $25 \mathrm{yr}$, range 18-36) participated in the experiment, which was performed according to the guidelines provided by the local medical ethics committee. The experiment consisted of five study-test cycles, whereby every four subjects saw the same set of five study-test cycles. As stimuli, 360 grayscale photographs of natural landscapes with or without buildings were randomly divided into five sets of pictures, 36 with and 36 without buildings. During the study, stimuli were colored in a light, lurid, and dark shade of red or green. During testing, stimuli were shown in grayscale (for an overview of the experimental set-up, see Fig. 3).

During each of the study phases, 48 photographs were displayed for $1400 \mathrm{msec}$, followed by a fixation point appearing for 2400-3700 msec. Following each photograph, a screen with the three different shades of either red or green squares (depending on the color of the antecedent picture) (Fig. 3) appeared for 1200 msec. During each of the test phases, the 48 old photographs were presented intermixed with 24 new ones, each for 1400 msec, followed by a jittered fixation period between $2400-3700$ msec. To optimize the event sequence for statistical analysis, jittering of the interstimulus interval was done as indicated before, and 24 null-events (black screen with a fixation point in the middle for $2000 \mathrm{msec}$ ) during study and 36 null-events during tests were intermixed.

At study, subjects memorized each picture together with its color/shade by making a landscape/building (covertly) and a color/shade-decision (button press). During retrieval, the subjects were required to indicate by a button press whether the displayed photograph either was not seen before ("new") or was seen at study and previously presented in red ("old previously red") or green ("old previously green"). After a "new" judgment, no further action was required for this particular item. If the subject classified the photograph to be old previously red or old previously green, three color-cues with the three different shades of either red or green were shown, and subjects were required to decide in which shade the photograph had been presented during the study phase (see Fig. 3). In keeping with previous studies (Weis et al. $2004 \mathrm{a}, \mathrm{b}$ ), for old/new and shade decisions, subjects could make use of an "uncertain" response in order to avoid a contamination by guesses. The use of the left or right hand for button presses was counterbalanced across subjects. Before scanning, a practice session was given to the participant to ensure that the subject was familiar with the entire procedure in the scanner.

Functional MR volumes were acquired with an ascending slice-acquisition $\mathrm{T} 2{ }^{*}$-weighted echo-planar imaging sequence
Study

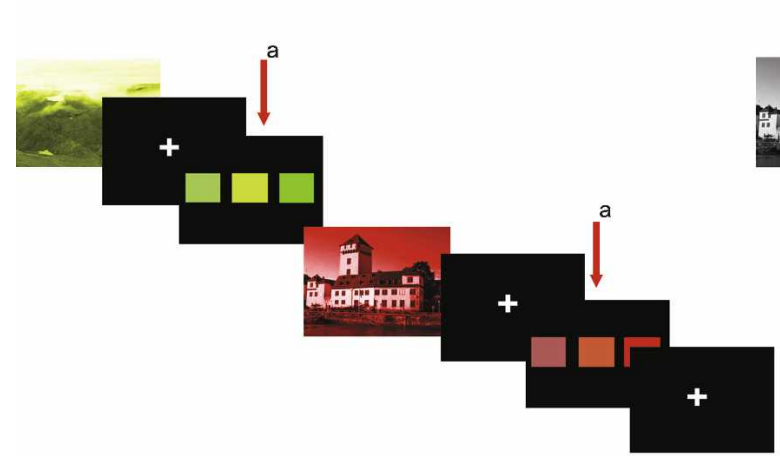

Test

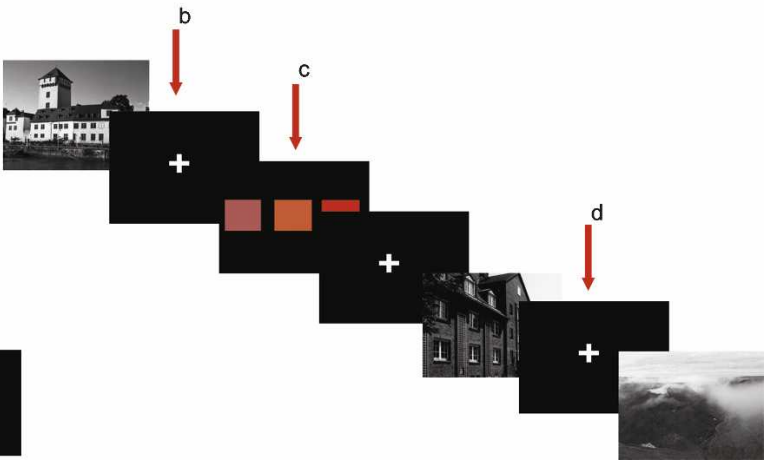

Figure 3. Simplified depiction of study and test trials. The letters a-d indicate whenever a button press had to be made. At a, subjects were required to indicate during study which color and shade of the color the pictures were dyed in. At b, subjects judged by button presses whether the photo was previously presented in red (old, previously red) or in green (old, previously green). If the subject judged the photograph to be old, previously red or old, previously green, subjects were required to indicate, at $c$, in which shade the photograph had been presented during the study phase. At $d$, subjects were required to press the button if they had not seen the photo before (new). 
(Sonata $1.5 \mathrm{~T}$, Siemens; 33 axial slices; volume repetition time $[\mathrm{TR}]=2.29 \mathrm{sec}$; echo time $[\mathrm{TE}]=30 \mathrm{msec} ; 90^{\circ}$ flip angle; slice matrix $=64 \times 64$; slice thickness $=3.0 \mathrm{~mm}$; slice gap $=0.5 \mathrm{~mm}$; isotropic voxel size $=3.5 \times 3.5 \times 3.5 \mathrm{~mm}^{3}$; field of view $=224 \mathrm{~mm}$ ) during encoding and retrieval of the present experiment. A T1weighted MP-RAGE sequence (176 sagittal slices; volume TR $=2250$ msec; $\mathrm{TE}=3.93 \mathrm{msec} ; 15^{\circ}$ flip angle; slice matrix $=256 \times 256$; slice thickness $=1.0 \mathrm{~mm}$; no gap; field of isotropic voxel size $=1 \times 1 \times 1$ $\mathrm{mm}^{3}$; view $=256 \mathrm{~mm}$ ) was acquired for anatomical localization.

Analysis was performed using SPM2 (Friston et al. 1995, 1996; www.fil.ion.ucl.ac.uk/spm). After discarding the first five volumes in every session to allow for T1 equilibration effects, the functional EPI-BOLD images were realigned, and the subject means were coregistered with the corresponding structural MR images using mutual information optimization. Volumes were subsequently slice-time corrected and then normalized to the stereotactic anatomical MNI space. Data were smoothed with an isotropic three-dimensional Gaussian kernel (8-mm FWHM). The fMRI data were analyzed statistically using the general linear model. The explanatory variables were temporally convolved with the canonical hemodynamic response function provided by SPM2. In addition, the linear model included a temporal highpass filter to account for various low-frequency effects and realignment parameters as effects of no interest. Relevant contrast parameter images were generated for each subject on the basis of the following test trials: (1) recognized old items, for which the correct color and shade was retrieved (2 source hits); (2) recognized items, for which only the correct color was retrieved (1 source hits); (3) recognized items without correct retrieval of contextual information (item hits); (4) old items misclassified as new (misses); and (5) correctly classified new items (correct rejections). Trial types 1,2, and 3 were combined as "overall hits." These contrasts were subsequently subjected to a second-level random effects analysis as outlined in the Results section. Note that the main purpose of this study was to dissociate MTL activity related to contextual and noncontextual memory retrieval so that a global analysis over the whole brain was only taken as a confirmation of significant activations that have been previously associated with contextual and noncontextual forms of recognition memory using a similar experimental set-up (Weis et al. 2004a,b). Therefore, we compared neural activity between all recognized items and misses as well as neural activity between all recognized items and new items over the whole brain thresholded at $P=0.001$. In this analysis, the cluster size, corrected for multiple nonindependent comparisons, was used as test statistic. To delineate the involvement of the MTL substructures, a subsequent region of interest (ROI) analysis was performed within the MTL using two anatomically defined mask-images generated for both left and right MTL (including hippocampus and parahippocampal and fusiform gyrus) based on the Talairach Daemon database (Maldjian et al. 2003). All $P$-values reported were corrected for multiple nonindependent comparisons (small volume correction based on the family-wise error). We report MNI $X / Y / Z$ coordinates of local maxima together with the voxel and corresponding volumina size of the significant cluster.

Two kinds of ROI analyses were performed within the MTL. In the first analysis, we analyzed by means of $t$-tests whether there were any significant differences between hits with correct retrieval of context (collapsed over 1 source hits and 2 source hits) and hits without correct retrieval of context (item hits) as well as significant differences between hits with correct retrieval of context (collapsed over 1 source hits and 2 source hits) and correctly classified new items (correct rejections). This analysis also included a comparison of activity decrease and increase between misses and item hits to further dissociate neural activity related to mere item recognition (Henson et al. 2003, 2005; Weis et al. 2004a; Gonsalves et al. 2005; Daselaar et al. 2006; Montaldi et al. 2006).

Second, we conducted a linearly weighted analysis investigating how the activation pattern of MTL substructures changes as a function of memory strength across the different conditions of previously presented stimuli, assuming that correctly recognized items for which the two contextual details could be recalled, yielded the strongest memory while misses should yield the weakest memory.

\section{Acknowledgment}

We thank Paul Gaalman for his technical assistance in acquiring the MRI data.

\section{References}

Bowles, B., Crupi, C., Mirsattari, S.M., Pigott, S.E., Parrent, A.G., Pruessner, J.C., Yonelinas, A.P., and Köhler, S. 2007. Impaired familiarity with preserved recollection after anterior temporal-lobe resection that spares the hippocampus. Proc. Natl. Acad. Sci. 104: 16382-16387.

Boynton, G.M., Engel, S.A., Glover, G.H., and Heeger, D.J. 1996. Linear systems analysis of functional magnetic resonance imaging in human V1. J. Neurosci. 16: 4207-4221.

Brown, M.W. and Aggleton, J.P. 2001. Recognition memory: What are the roles of the perirhinal cortex and hippocampus? Nat. Rev. Neurosci. 2: 51-61.

Bunge, S.A., Burrows, B., and Wagner, A.D. 2004. Prefrontal and hippocampal contributions to visual associative recognition: Interactions between cognitive control and episodic retrieval. Brain Cogn. 56: 141-152.

Cansino, S., Maquet, P., Dolan, R.J., and Rugg, M.D. 2002. Brain activity underlying encoding and retrieval of source memory. Cereb. Cortex 12: $1048-1056$.

Daselaar, S.M., Fleck, M.S., and Cabeza, R. 2006. Triple dissociation in the medial temporal lobes: Recollection, familiarity, and novelty. J. Neurophysiol. 96: 1902-1911.

Davachi, L. 2006. Item, context, and relational episodic encoding in humans. Curr. Opin. Neurobiol. 16: 693-700.

Diana, R.A., Yonelinas, A.P., and Ranganath, C. 2007. Imaging recollection and familiarity in the medial temporal lobe: A three-component model. Trends Cogn. Sci. 11: 379-386.

Dobbins, I.G., Rice, H.J., Wagner, A.D., and Schacter, D.L. 2003. Memory orientation and success: Separable neurocognitive components underlying episodic recognition. Neuropsychologia 41: $318-333$.

Eichenbaum, H. and Cohen, N.J. 2001. From conditioning to conscious recollection: Memory systems of the brain. Oxford University Press, Oxford, UK.

Eichenbaum, H., Yonelinas, A.P., and Ranganath, C. 2007. The medial temporal lobe and recognition memory. Annu. Rev. Neurosci. 30: $123-152$.

Eldridge, L.L., Knowlton, B.J., Furmanski, C.S., Bookheimer, S.Y., and Engel, S.A. 2000. Remembering episodes: A selective role for the hippocampus during retrieval. Nat. Neurosci. 3: 1149-1152.

Fernández, G. and Tendolkar, I. 2006. The rhinal cortex: "Gatekeeper" of the declarative memory system. Trends Cogn. Sci. 10: 358-362.

Friston, K.J., Holmes, A.P., Poline, J.B., Grasby, P.J., Williams, S.C., Frackowiak, R.S., and Turner, R. 1995. Analysis of fMRI time-series revisited. Neuroimage 2: 45-53.

Friston, K.J., Homes, A.P., Pline, J.-B., Price, C.J., and Frith, C.D. 1996. Detecting activations in PET and fMRI: Levels of inference and power. Neuroimage 4: 223-235.

Giovanello, K.S., Schnyer, D.M., and Verfaellie, M. 2004. A critical role for the anterior hippocampus in relational memory: Evidence from an fMRI study comparing associative and item recognition. Hippocampus 14: 5-8.

Gonsalves, B.D., Kahn, I., Curran, T., Norman, K.A., and Wagner, A.D. 2005. Memory strength and repetition suppression: Multimodal imaging of medial temporal cortical contributions to recognition. Neuron 47: 751-761.

Hayes, S.M., Nadel, L., and Ryan, L. 2007. The effect of scene context on episodic object recognition: Parahippocampal cortex mediates memory encoding and retrieval success. Hippocampus 17: 873-889.

Henson, R.N., Cansino, S., Herron, J.E., Robb, W.G., and Rugg, M.D. 2003. A familiarity signal in human anterior medial temporal cortex? Hippocampus 13: 301-304.

Henson, R.N., Hornberger, M., and Rugg, M.D. 2005. Further dissociating the processes involved in recognition memory: An FMRI study. J. Cogn. Neurosci. 17: 1058-1073.

Holdstock, J.S., Mayes, A.R., Gong, Q.Y., Roberts, N., and Kapur, N. 2005. Item recognition is less impaired than recall and associative recognition in a patient with selective hippocampal damage. Hippocampus 15: 203-215.

Hornberger, M., Rugg, M.D., and Henson, R.N. 2006. fMRI correlates of retrieval orientation. Neuropsychologia 44: 1425-1436.

Insausti, R., Amaral, D.G., and Cowan, W.M. 1987. The entorhinal cortex of the monkey: II. Cortical afferents. J. Comp. Neurol. 264: 356-395.

Kirwan, C.B. and Stark, C.E. 2004. Medial temporal lobe activation during encoding and retrieval of novel face-name pairs. Hippocampus 
14: 9191-9230.

Maldjian, J.A., Laurienti, P.J., Kraft, R.A., and Burdette, J.H. 2003. An automated method for neuroanatomic and cytoarchitectonic atlas-based interrogation of fMRI data sets. Neuroimage 19: $1233-1239$.

Mandler, G. 1980. Recognising: The judgement of previous occurrence. Psychol. Rev. 87: 252-271.

Manns, J.R., Hopkins, R.O., Reed, J.M., Kitchener, E.G., and Squire, L.R. 2003. Recognition memory and the human hippocampus. Neuron 37: 171-180.

Meltzer, J.A. and Constable, R.T. 2005. Activation of human hippocampal formation reflects success in both encoding and cued recall of paired associates. Neuroimage 24: 384-397.

Montaldi, D., Spencer, T.J., Roberts, N., and Mayes, A.R. 2006. The neural system that mediates familiarity memory. Hippocampus 16: $504-520$.

Moser, M.B. and Moser, E.I. 1998. Functional differentiation in the hippocampus. Hippocampus 8: 608-619.

O'Reilly, R.C. and Norman, K.A. 2002. Hippocampal and neocortical contributions to memory: Advances in the complementary learning systems framework. Trends Cogn. Sci. 6: 505-510.

Sauvage, M.M., Fortin, N.J., Owens, C.B., Yonelinas, A.P., and Eichenbaum, H. 2008. Recognition memory: Opposite effects of hippocampal damage on recollection and familiarity. Nat. Neurosci. 11: $16-18$.

Scoville, W.B. and Milner, B. 1957. Loss of recent memory after bilateral hippocampal lesions. J. Neurol. Neurosurg. Psychiatr. 20: 11-21.

Soltysik, D.A., Peck, K.K., White, K.D., Crosson, B., and Briggs, R.W. 2004. Comparison of hemodynamic response nonlinearity across primary cortical areas. Neuroimage 22: 1117-1127.

Squire, L.R., Stark, C.E., and Clark, R.E. 2004. The medial temporal lobe. Annu. Rev. Neurosci. 27: 279-306.

Squire, L.R., Wixted, J.T., and Clark, R.E. 2007. Recognition memory and the medial temporal lobe: A new perspective. Nat. Rev. Neurosci. 8: $872-883$.

Stark, C.E. and Squire, L.R. 2003. Hippocampal damage equally impairs memory for single items and memory for conjunctions. Hippocampus 13: $281-292$.

Suzuki, W.A. and Amaral, D.G. 1994. Perirhinal and parahippocampal cortices of the macaque monkey: Cortical afferents. J. Comp. Neurol. 350: $497-533$.

Tendolkar, I., Arnold, J., Petersson, K.M., Weis, S., Brockhaus-Dumke, A., van Eijndhoven, P., Buitelaar, J., and Fernández, G. 2007. Probing the neural correlates of associative memory formation: A parametrically analyzed event-related functional MRI study. Brain Res. 1142: 159-168.

Wais, P.E., Wixted, J.T., Hopkins, R.O., and Squire, L.R. 2006. The hippocampus supports both the recollection and the familiarity components of recognition memory. Neuron 49: 459-466.

Weis, S., Specht, K., Klaver, P., Tendolkar, I., Willmes, K., Ruhlmann, J., Elger, C.E., and Fernández, G. 2004a. Process dissociation between contextual retrieval and item recognition. Neuroreport 15: 2729-2733.

Weis, S., Klaver, P., Reul, J., Elger, C.E., and Fernández, G. 2004b. Neural correlates of successful declarative memory formation and retrieval: The anatomical overlap. Cereb. Cortex 40: 200-202.

Yonelinas, A.P., Hopfinger, J.B., Buonocore, M.H., Kroll, N.E., and Baynes, K. 2001. Hippocampal, parahippocampal and occipital-temporal contributions to associative and item recognition memory: An fMRI study. Neuroreport 12: 359-363.

Yonelinas, A.P., Otten, L.J., Shaw, K.N., and Rugg, M.D. 2005. Separating the brain regions involved in recollection and familiarity in recognition memory. J. Neurosci. 25: 3002-3008.

Received January 18, 2008; accepted in revised form June 9, 2008. 


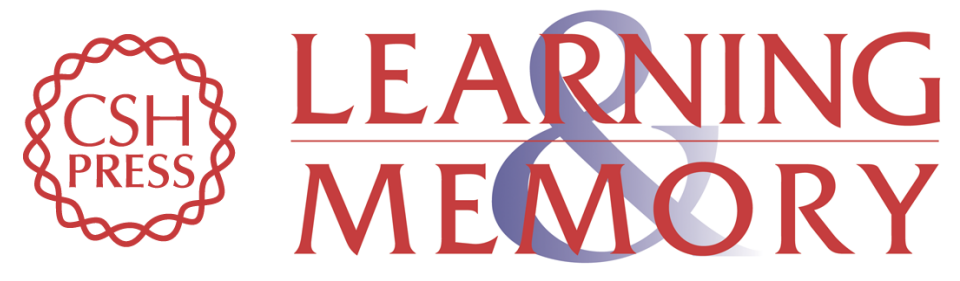

\section{Contributions of the medial temporal lobe to declarative memory retrieval: Manipulating the amount of contextual retrieval}

Indira Tendolkar, Jennifer Arnold, Karl Magnus Petersson, et al.

Learn. Mem. 2008, 15:

Access the most recent version at doi:10.1101//m.916708

References This article cites 43 articles, 4 of which can be accessed free at:

http://learnmem.cshlp.org/content/15/9/611.full.html\#ref-list-1

License

Email Alerting Receive free email alerts when new articles cite this article - sign up in the box at the Service top right corner of the article or click here. 